\title{
Highly efficient near-infrared hybrid organic-inorganic nanocrystal electroluminescence device
}

\author{
K. N. Bourdakos, ${ }^{1}$ D. M. N. M. Dissanayake, ${ }^{2}$ T. Lutz, ${ }^{2}$ S. R. P. Silva, ${ }^{2}$ and R. J. Curry ${ }^{2, a)}$ \\ ${ }^{1}$ Department of Physics, University of Durham, South Road, Durham DH1 3 LE, United Kingdom \\ ${ }^{2}$ Nano-Electronics Centre, Advanced Technology Institute, University of Surrey, Guildford, \\ Surrey GU2 7XH, United Kingdom
}

(Received 1 February 2008; accepted 25 March 2008; published online 18 April 2008)

\begin{abstract}
We report the use of PbS nanocrystals within a hybrid device that emits $1.2 \mu \mathrm{m}$ electroluminescence with an external quantum efficiency of $1.15 \%$ corresponding to an internal quantum efficiency of $\sim 5 \%-12 \%$ thus demonstrating a viable, low-cost, highly efficient near infrared organic electroluminescent device. Direct generation of the excited state on the nanocrystal result in eliminating competing processes that have previously led to the low reported efficiencies in near-infrared light emitting devices. Furthermore, the emission wavelength can be tuned to cover a wide range of wavelengths including the $1.3-1.5 \mu \mathrm{m}$ region without significant change of the efficiency. () 2008 American Institute of Physics. [DOI: 10.1063/1.2909589]
\end{abstract}

Organic molecules are generally optically active in the range of wavelengths below $1 \mu \mathrm{m}$ due to the fundamental properties of delocalized $\pi$ systems. Even when modified through substitution, or ligation of heavy metal atoms, significant extension of optical activity beyond $1 \mu \mathrm{m}$ is difficult. Near-infrared (NIR) organic/polymer devices emitting electroluminescence (EL) above $1 \mu \mathrm{m}$ would have a number applications including (bio)sensing, waveguide and free space chip-to-chip optical circuits, and telecommunications. These factors have driven significant research into extending the optical activity of organic materials into this wavelength region despite the inherent difficulties.

The most studied approach has been the use of organolanthanide complexes in which intra-atomic $4 f-4 f$ transitions are used to obtain characteristic narrow emission in the NIR from ions such as $\mathrm{Er}^{3+}$ and $\mathrm{Nd}^{3+} \cdot{ }^{1-3}$ However, devices based on this concept have proved to be highly inefficient $(<0.01 \%)$ due to poor excitation of the lanthanide ion and strong competing nonradiative relaxation mechanisms. ${ }^{4,5}$

The development of colloidal quantum dots consisting of semiconductor nanocrystals $^{6-8}$ has provided an alternative approach toward obtaining a low cost NIR electroluminescent device. The ability to directly modify the bandgap through selection of the nanocrystal material and diameter allows for emission to be obtained from the visible and through the NIR region. The incorporation of nanocrystals within organic devices as the emitting center allows low-cost solution processing to be used and direct access to the NIR region. To date, the highest reported external quantum efficiency (EQE) from such hybrid devices are $0.5 \%$ (Ref. 9) (emitting $\sim 1.3 \mu \mathrm{m}$ ) and $0.27 \%$ (Ref. 10) (emitting $\sim 1.15 \mu \mathrm{m}$ ) utilizing a MEH-PPV-PbS and MEH-PPV-InAs composite architecture. In these devices, the excited nanocrystal state is achieved through exciton transfer from nearby molecules. As a result, the quantum efficiency relating to emission originating from the nanocrystals is critically dependent upon this energy transfer step which must compete against radiative and nonradiative recombination of excitons prior to energy transfer. Given the short range dependence of

${ }^{a)}$ Electronic mail: r.j.curry@surrey.ac. uk.
Förster or Dexter energy transfer, systems that rely upon these methods to excite the nanocrystals are likely to be inefficient NIR emitters and limited by this step. This is evident as emission is often observed in the visible region originating from excitons radiatively recombining on the organic or polymer host where they were created. ${ }^{9}$ Nonradiative effects will also reduce the population of host excitons available for energy transfer to nanocrystals in these systems including, for example, phonon scattering and multiexciton annihilation.

In this work, we describe the fabrication of a NIR electroluminescent device in which excitons are directly created on the nanocrystals thus removing the requirement for exciton generation on organic molecules and subsequent transfer to the nanocrystals. In this simplified device architecture the ability to optimize of the parameters to enhance device efficiency is significantly increased. The reported devices exploit a layer of $\mathrm{PbS}$ nanocrystals (nc-PbS) and a layer of pentacene. The hybrid nc-PbS organic light-emitting diode operates at the peak wavelength of $\sim 1.2 \mu \mathrm{m}$ with an EQE of $>1.1 \%$ corresponding to an internal quantum efficiency (CIQE) in the range of $5 \%-12 \%$, based on previous reports, ${ }^{9,10}$ and thus representing a significant breakthrough in the field.

Oleic acid capped nc-PbS were synthesized according to the method described elsewhere ${ }^{11}$ with ligand exchange performed $^{12}$ to provide butylamine ligands. This involved drying the nc-PbS under nitrogen followed by resuspension using ultrasonication in an excess of butylamine (Fluka, 99.5\%). The flask was sealed under nitrogen and stirred for $72 \mathrm{~h}$ following which the dots were precipitated out using degassed isopropanol. Centrifugation at $5000 \mathrm{rpm}$ was then carried out and the supernatant dried under nitrogen leading to a powder of nc-PbS that was subsequently dissolved in toluene at a concentration of $50 \mathrm{mg} / \mathrm{ml}$.

Devices were fabricated in a manner similar to that previously reported for organic-nanocrystal photovoltaic devices. $^{13}$ Indium tin oxide (ITV) $(20 \Omega / \square)$ was cleaned prior to use including oxygen plasma treatment. Pentacene (H.W. Sands, 99.99\%) was used as received and 2,9dimethyl-4,7-diphenyl-1,10-phenanthroline (BCP) (Aldrich 


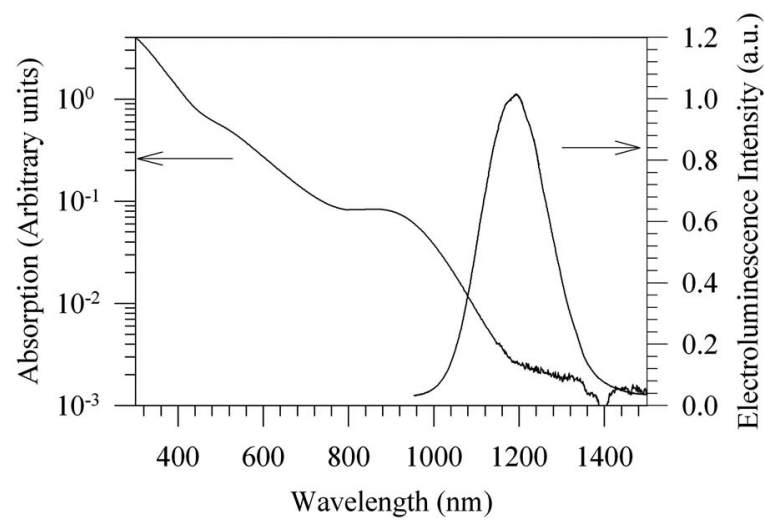

FIG. 1. The absorption nc-PbS and EL spectrum of ITO/pentacene/nc-PbS/ $\mathrm{BCP} / \mathrm{Al}$ devices. The absorption spectrum was obtained from nc-PbS capped with butylamine ligands dissolved in toluene $(50 \mathrm{mg} / \mathrm{ml})$.

98\%) was twice sublimated prior to use. The basic device structure is $\mathrm{ITO} /$ pentacene $(50 \mathrm{~nm}) / \mathrm{nc}-\mathrm{PbS}(110 \mathrm{~nm}) / \mathrm{BCP}$ $(10 \mathrm{~nm}) / \mathrm{Al}(60 \mathrm{~nm})$ with an active device area of $\sim 7 \mathrm{~mm}^{2}$.

Absorption measurements were carried out using a Varian Cary 5000 spectrophotometer. A Kethley 2400 source-measure unit was used to obtain current-voltage measurements and the EL directly collected and dispersed in a Jobin Yvon Horriba iHr 550 spectrometer with a $150 \mathrm{gr} / \mathrm{mm}$ grating blazed at $1200 \mathrm{~nm}$ onto a 512 element front electrode liquid $\mathrm{N}_{2}$ cooled InGaAs array (Symphony IGA) or $\mathrm{Si}$ charge-copled device $(1024 \times 512$ pixels, Symphony). The $\mathrm{EQE}$ of the device was determined using integrated EL measurements performed using the same voltage source and an InGaAs OMH 6727B integrating sphere with a $5 \mathrm{~mm}$ diameter entrance aperture. The device was placed at about $2 \mathrm{~mm}$ distance (due to the thickness of the ITO-glass slide and mounting glass slide used to hold the device) from the aperture of the integrating sphere, thus, assuming Lambertian emission, $\sim 99 \%$ of light emitted from the planar substrate surface was collected.

Figure 1 shows the absorption of the $\mathrm{nc}-\mathrm{PbS}$ in solution and the EL spectrum emitted by the device. The $1 s_{e}-1 s_{h}$ absorption peak of the nc-PbS can be seen to be centered at $\sim 900 \mathrm{~nm}$. The feature at $\sim 1400 \mathrm{~nm}$ in the absorption spectrum is an artifact of the measurement system. Strong EL is obtained from the device peaking at $\sim 1200 \mathrm{~nm}$ and can be fitted with a Gaussian curve with width of $100 \mathrm{~nm}$. The broad width of the emission is due to the inhomogeneous broadening caused by the size distribution of the nc- $\mathrm{PbS}$ in the sample used. ${ }^{10}$ EL spectra were measured over the $550-1600 \mathrm{~nm}$ spectral range with no other emission (e.g., from pentacene) being detected, hence all optical power measured when determining the external $\mathrm{EQE}$ from the $\mathrm{nc}-\mathrm{PbS}$.

Figure 2 shows the current density versus voltage $(J-V)$ and luminescence versus voltage $(L-V)$ characteristics of the device. The device shows excellent rectification characteristics with the turn on voltage observed to be $\sim 1 \mathrm{~V}$ and reverse bias break down occurring at $\sim-6 \mathrm{~V}$. Hole injection occurs into the highest occupied molecular orbital of pentacene $(4.9 \mathrm{eV})$ (Ref. 14) from the Fermi level of ITO which was previously measured to be $4.7 \mathrm{eV} .{ }^{15}$ Electron injection occurs through the BCP layer, aided by the presence of interfacial states, into the nc-PbS. There is a large barrier for electron transfer from the nc-PbS into the pentacene [lowest Downloaded 30 Mar 2009 to 131.227.178.132. Redistribution subje

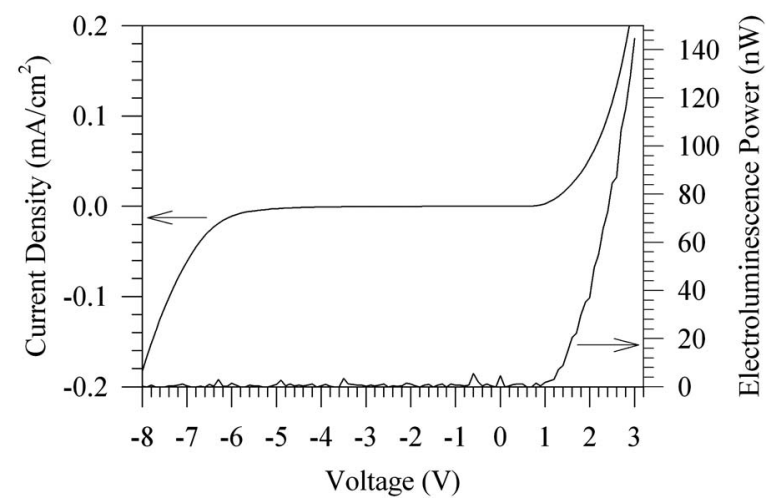

FIG. 2. The current density-voltage $(J-V)$ and luminescence-voltage $(L-V)$ characteristics of the ITO/pentacene/nc- $\mathrm{PbS} / \mathrm{BCP} / \mathrm{Al}$ devices.

unoccupied molecular orbital $3.0 \mathrm{eV}$ (Ref. 14)] based on previously reported values of the nc-PbS energy levels (conduction band $\sim 4.7 \mathrm{eV}) .{ }^{13}$ Following hole injection, exciton formation occurs within nc-PbS which are prevented from quenching at the $\mathrm{Al}$ electrode due to the presence of the wide bandgap BCP layer. We believe that the turn-on voltage $(\sim 1 \mathrm{~V})$ is mainly a result of the barrier for hole injection into the nc-PbS. The presence of any interfacial dipole between the pentacene and nc-PbS as well as any internal dipole of the nc-PbS will also be influential in determining the voltage required for hole injection from the pentacene. The large Stokes shift observed $(\sim 300 \mathrm{~nm})$ between the $\mathrm{nc}-\mathrm{PbS}$ absorption and electroluminescent spectra supports the presence of a barrier to hole injection from the pentacene into the $\mathrm{nc}-\mathrm{PbS}$ being present. Such a barrier would be reduced for smaller bandgap nc-PbS thus preferential injection into these smaller bandgap nc-PbS may occur resulting in the large Stokes shift. This will be the focus of further investigation in the near future.

Figure 3 shows direct measurements of the device EQE indicating a peak value of $1.15 \%$ at $1.6 \mathrm{~V}$ representing a significant improvement over previously reported devices. We also have directly measured the EQE using integrating spectroscopic methods thus removing the need to calculate the EQE from the EL spectra for example. Device operation at an EQE above $1 \%$ is seen across a wide operating voltage range of $1.5-2.5 \mathrm{~V}$ and only dropping slightly to $\sim 0.85 \%$ at $3 \mathrm{~V}$ still far exceeding all previous work in this field. Each of these measurements presented is the average of three measurements taken at the same voltage over a period of $\sim 90 \mathrm{~s}$.

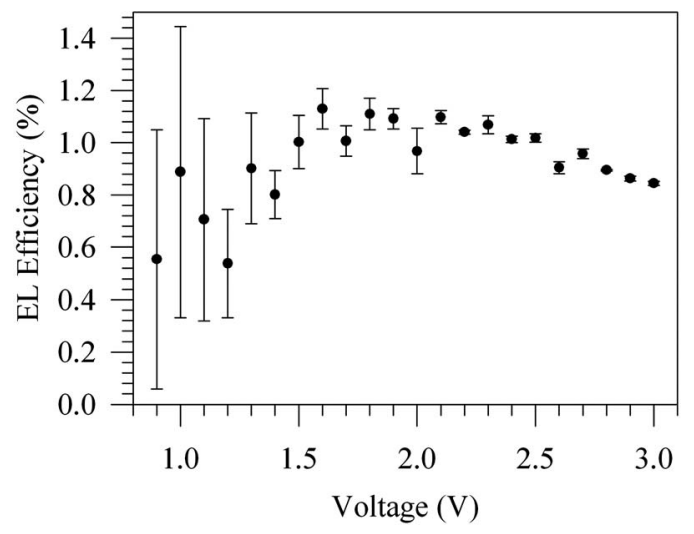

FIG. 3. EQE of the ITO/pentacene/nc- $\mathrm{PbS} / \mathrm{BCP} /$ devices as a function of voltage.

to AIP license or copyright; see http://apl.aip.org/apl/copyright.jsp 
These high values of EQE are directly related to the low current densities within these devices. This is a consequence of the device design utilized and the direct creation of excitons on the $\mathrm{nc}-\mathrm{PbS}$ removing competing mechanisms relating to the need for exciton transfer as discussed above, and any visible emission from other species within the devices.

Previous reports have estimated the IQE of nanocrystal based devices to have a value ranging from three to seven times the EQE (Refs. 9 and 10) with nc-PbS based devices being at the top end of this range. Applying a similar approach to the devices reported within and taking into account the $70 \%$ transmission of the ITO/glass substrate, we obtain values of IQE ranging from $5 \%$ to $12 \%$. These values of $\mathrm{EQE}$ and IQE represent a significant breakthrough in the development of organic diode NIR sources. Given the operating wavelengths of these devices, the excitation mechanism, the exceptionally low power consumption and previously demonstrated $\mathrm{Si}$ compatibility, ${ }^{16}$ we believe this work will enable the development of devices that will impact on a wide variety of application areas.

In summary, we have demonstrated efficient NIR EL from a hybrid organic-inorganic devices emitting at $1.2 \mu \mathrm{m}$ using nc-PbS as the emitting center. These devices represent a significant improvement in the state of the art and offer the potential for the development of low-cost NIR devices.
${ }^{1}$ R. J. Curry and W. P. Gillin, Appl. Phys. Lett. 75, 1380 (1999).

${ }^{2}$ R. J. Curry, M. Somerton, and W. P. Gillin, J. Adhes. 88, 777 (2000).

${ }^{3}$ L. H. Sloof, A. Polman, F. Cacialli, R. H. Friend, G. A. Hebbonk, F. C. J.

M. van Veggel, and D. N. Reinhoudt, Appl. Phys. Lett. 78, 2122 (2001).

${ }^{4}$ R. J. Curry, W. P. Gillin, A. P. Knights, and R. Gwilliam, Opt. Mater. (Amsterdam, Neth.) 17, 165 (2001).

${ }^{5}$ B. S. Harrison, T. J. Foley, M. Bouguettaya, J. M. Boncella, J. R. Reynolds, K. S. Schanze, J. Shim, P. H. Holloway, G. Padmanaban, and S. Ramakrishnan, Appl. Phys. Lett. 79, 3770 (2001).

${ }^{6}$ M. L. Steigerwald, A. P. Alivisatos, J. M. Gibson, T. D. Harris, R. Kortan, A. J. Muller, A. M. Thayert, T. M. Duncan, D. C. Douglass, and L. E. Brus, J. Am. Chem. Soc. 110, 3046 (1988).

${ }^{7}$ C. B. Murray, D. B. Norris, and M. G. Bawendi, J. Am. Chem. Soc. 115, 8706 (1993).

${ }^{8}$ M. A. Hines and G. D. Scholes, Adv. Mater. (Weinheim, Ger.) 15, 1844 (2003).

${ }^{9}$ N. Tessler, V. Medvedev, M. Kazes, S. Kan, and U. Banin, Science 295, 1506 (2002).

${ }^{10}$ G. Konstantatos, C. Huang, L. Levina, Z. Lu, and E. H. Sargent, Adv. Funct. Mater. 15, 1865 (2005).

${ }^{11}$ M. A. Hines and G. D. Scholes, Adv. Mater. (Weinheim, Ger.) 15, 1844 (2003).

${ }^{12}$ G. Konstantatos, I. Howard, A. Fischer, S. Hoogland, J. Clifford, E. Klem, L. Levina, and E. H. Sargent, Nature (London) 442, 180 (2006).

${ }^{13}$ D. M. N. M. Dissanayake, R. A. Hatton, T. Lutz, C. E. Giusca, R. J. Curry, and S. R. P. Silva, Appl. Phys. Lett. 91, 133506 (2007).

${ }^{14}$ S. Yoo, B. Domercq, and B. Kippelen, Appl. Phys. Lett. 85, 5427 (2004).

${ }^{15}$ A. J. Miller, R. A. Hatton, and S. R. P. Silva, Appl. Phys. Lett. 89, 123115 (2006).

${ }^{16}$ R. J. Curry, W. P. Gillin, A. Knights, and R. Gwilliam, Appl. Phys. Lett. 77, 2271 (2000) 